\title{
Focal Cortical Dysplasia
}

Peter B. Crino, MD, $\mathrm{PhD}^{1}$

${ }^{1}$ Department of Neurology, Shriners Hospital Pediatric Research Center and Temple University, Philadelphia, Pennsylvania

Address for correspondence Peter B. Crino, MD, PhD, Department of Neurology, Shriners Hospitals Pediatric Research Center, Temple University School of Medicine, 6th Floor Medical Education and Research Building, 3500 N. Broad Street, Philadelphia, PA 19140-4106 Semin Neurol 2015;35:201-208.

(e-mail: peter.crino@temple.edu).

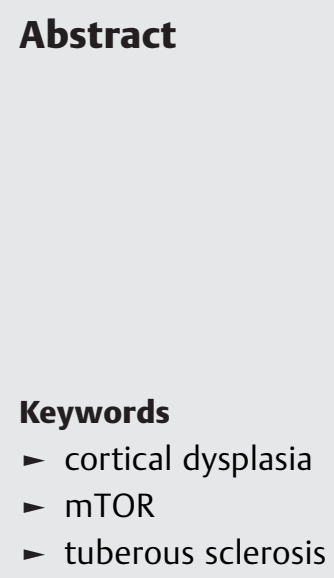

Focal cortical dysplasias are common malformations of cerebral cortical development and are highly associated with medically intractable epilepsy. They have been classified into neuropathological subtypes (type la, Ib, Ila, Ilb, and III) based on the severity of cytoarchitectural disruption-tangential or radial dispersion, or loss of laminar structure -and the presence of unique cells types such as cytomegalic neurons or balloon cells. Most focal cortical dysplasias can be identified on neuroimaging and many require resective epilepsy surgery to cure refractory seizures. The pathogenesis of focal cortical dysplasias remains to be defined, although there is recent evidence to suggest that focal cortical dysplasias arise from de novo somatic mutations occurring during brain development. Some focal cortical dysplasia subtypes show a link to the mammalian target of rapamycin signaling cascade; this has now extended to other cortical malformations, including hemimegalencephaly.
Focal cortical dysplasias (FCDs) comprise a spectrum of focal developmental malformations characterized by disruption of the normal cytoarchitecture of the cerebral cortex. They are highly associated with medically intractable epilepsy. ${ }^{1-3}$ Focal cortical dysplasia and epilepsy were first associated in a report by Taylor $^{4}$ (although previously alluded to by Crome $^{5}$ ), who reported 10 patients (adults and children) with refractory epilepsy undergoing surgical resection, who showed focal abnormalities of cortical cytoarchitecture that matched the proposed anatomical focus associated with their seizure semiology. They hypothesized that the focal pathological changes were probably developmental and that they accounted for their seizures. The description of other focal malformations of cortical development (MCD) subtypes sharing pathological changes with FCD-such as hemimegalencephaly ${ }^{6}$ and tuberous sclerosis complex (TSC) ${ }^{7}$-dates back to the 1800s. There have also been recent descriptions of new FCD syndromes, including Pretzel syndrome, autosomal dominant temporal lobe epilepsy and cortical dysplasia, and familial focal epilepsy with variable foci (see below).

\section{Classification and Neuropathology}

Historically, several different FCD classification systems have been proposed, ${ }^{8-10}$ trying to link the pathological findings with developmental mechanisms, although none has consistently linked the pathology to the clinical presentation or outcome. Distinct classification schemes have been proposed to define the relevant imaging and histological features of FCD. ${ }^{8}$ The Palmini classification system ${ }^{9}$ was restructured and further subdivided FCD into type IA, IB, IIA, and IIC. The International League Against Epilepsy (ILAE) task force of the Diagnostic Methods Commission generated a new consensus classification of distinct focal cortical dysplasia subtypes based on histopathological features ${ }^{10}$ that yields consistent interobserver and intraobserver reliability. The ILAE classification scheme comprises a three-tiered system, including both isolated and associated FCD variants. A new and comprehensive classification scheme assumes that all MCD types result from distinct developmental and molecular genetic causes, and that these directly affect cortical development at distinct epochs and within distinct cell types. ${ }^{11}$

Focal cortical dysplasia type I is characterized by abnormal cortical layering with radial microcolumns, and is of three subtypes. Focal cortical dysplasia type la shows radial microcolumns resembling the microcolumnar organization pattern of the early stages of cortical development; FCD type Ib shows tangential layer alterations; FCD type Ic shows a combination of both. All the three variants can show heterotopic neurons in white matter and hypertrophic neurons (outside layer 5),
Issue Theme Etiology of Epilepsy; Guest Editors: Philip Smith, MD, FRCP,

FAcadMEd, and Rhys Thomas, BSc, MRCP, MSc, PhD
Copyright $\odot 2015$ by Thieme Medical Publishers, Inc., 333 Seventh Avenue, New York, NY 10001, USA. Tel: +1(212) 584-4662.
DOI http://dx.doi.org/ 10.1055/s-0035-1552617. ISSN 0271-8235. 
as well as normal neurons with abnormal dendrites. Focal cortical dysplasia type I may affect one or multiple lobes, yet preoperative magnetic resonance imaging (MRI) may be normal. Focal cortical dysplasia type I may be subtle and challenging to detect on routine neuropathology, but should be specifically sought in cases with normal preoperative MRI scans.

Focal cortical dysplasia type II is common among epilepsy surgical series and is a major cause of antiepileptic drugresistant epilepsy. It is more common in extratemporal areas, particularly in the frontal lobe, and is typically seen on preoperative MRI scans. Focal cortical dysplasia type IIa is characterized by dysmorphic and cytomegalic neurons, but lacking balloon cells; FCD Type IIb is characterized by dysmorphic/cytomegalic neurons and balloon cells. Balloon cells have an enlarged cell body and opalescent, glassy appearing, eosinophilic cytoplasm ( - Fig. 1). Cortical tubers are a type of FCD found in TSC, though not included in the ILAE classification system, and occur as single or multiple lesions in more than $80 \%$ of patients with TSC. They are linked to both epilepsy and neurocognitive disabilities (for review, see ${ }^{12}$ ). Tubers are common in temporal and frontal regions and are characterized histopathologically by dyslamination, and heterogeneous cell types, such as dysmorphic neurons, reactive astrocytes, and so-called giant cells. Giant cells are histologically similar to the balloon cells found in FCD type IIb.

Both giant cells and balloon cells express proteins characteristic of neuroglial progenitor cells, such as SOX2, nestin, vimentin, and c-myc, suggesting a failure to differentiate before migration into the cortex..$^{13}$ The first study to examine lineage markers in FCD found that balloon cells and cytomegalic neurons expressed cell markers reflecting lineage derivation from the telencephalic ventricular zone, such as OTX-1 and MASH. ${ }^{14}$ A subsequent study in FCD I ${ }^{15}$ showed that FCD I specimens in younger patients characteristically have abnormal expression of Tbr1 and Otx1 in layer II, supporting their origins from radial glia; by contrast, FCDII showed distinct labeling of balloon cells (Pax6, ER81 and Otx1) and dysmorphic neurons (Tbr 1, N200, and Map1b), supporting their origins in intermediate progenitor cells. Tubers may be found as early as 20 weeks gestation, ${ }^{16-18}$ indicating that tubers (and by extension, focal cortical dysplasias) form during embryonic brain development, probably between weeks 10 and 20 of human gestation.

A significant advantage of the ILAE classification was that pathological changes adjacent to or associated with substantive brain lesions (such as vascular malformations and tumors) could be defined as FCD type III. The four different subtypes of FCD type III include IIIa, associated with hippocampal sclerosis; IIIb, associated with tumors; IIIc, associated with vascular malformations; and IIId, associated with any other lesion acquired during early life. Histopathologically, FCD type III subtypes show type I abnormalities, including altered cortical lamination. However, finding an abnormal band of small and clustered "granular" neurons in the outer part of layer II can distinguish FCDI type I from subtypes IIIad. ${ }^{19}$ Other types of focal MCD, such as TSC, hemimegalencephaly, and some of the newer focal cortical dysplasia syndromes have not yet been subsumed into the ILAE classification. However, cortical tubers are histologically similar to FCDIIb; hemimegalencephaly may occur both with and without balloon cells similar to FCDIIb; and familial focal epilepsy with variable features may show a "bottom-of-thesulcus" dysplasia with a type IIa or IIb phenotype. An important corollary to these classifications is that FCDs are often heterogeneous lesions with local variations in regional cytoarchitectural abnormalities. For example, some resected lesions may contain both type I and type FCD pathologies. From a diagnostic perspective, while pathologists describe the histological variations, they tend to use the most severe FCD subtype for final diagnosis.

\section{Clinical Presentation}

Epilepsy, often intractable, remains the most common clinical presentation for all types of FCD as well as TSC and hemimegalencephaly. ${ }^{1}$ Patients usually come to clinical attention with seizures in early childhood, although seizures may start at any age. In virtually all FCD subtypes, there is close concordance between the location of the seizure onset
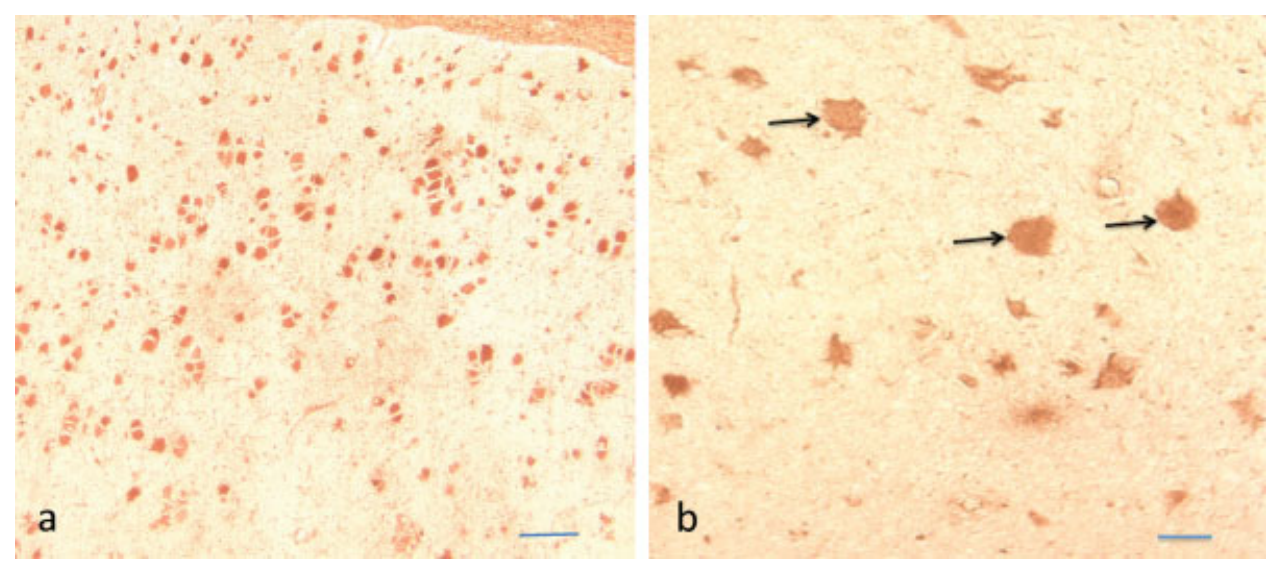

Fig. 1 (A) FCD type IIb (labeled with anti-nestin antibodies; modified from 14; scale bar $=400$ microns). Note loss of cortical lamination and ovoid shape of balloon cells. (B) Balloon cells (arrows) in cortical tuber (modified from 31; scale bar = 100 microns). 
defined by electroencephalography and the anatomic location of the FCD defined by MRI or intraoperative visualization. Furthermore, the anatomical location of FCD determines the seizure semiology. Frontal lobe epilepsy may manifest as sleep-related seizures characterized by stereotyped bilateral movements and vocalizations, sometimes with preserved consciousness. Patient with occipital lobe seizures may report visual symptoms, such as seeing dots or shapes in different colors. Regardless of semiology, recalcitrant and poorly controlled seizures contribute to cognitive impairment in children and adults. Other clinical presentations include developmental delay, behavioral issues, autism spectrum disorders, and sometimes focal neurologic deficits, depending on the size and location of the cortical lesion. The surgical outcome may vary with the pathological FCD subtype. ${ }^{20}$

\section{Radiographic Findings}

With greater availability of more advanced neuroimaging in the 1990s, specifically brain MRI, it became apparent that focal MCDs are more common in patients with intractable epilepsy than previously thought. Newer imaging shows MCD to be radiographically heterogeneous, with distinct signal characteristics, extent, and location. However, focal malformations of cortical development sometimes do not show on imaging, and can be found only on histopathological examination of resected tissue specimens. ${ }^{21}$ Nevertheless, neuroimaging is central to identifying and diagnosing FCDs. Common findings on brain MRI include increased cortical thickness, subtle changes in the smoothness of gyri or sulci, and changes in subcortical white matter signal. Typically, FCDs do not enhance with gadolinium, although approximately $5 \%$ of tubers in TSC enhance very slightly. In terms of radiographic-pathologic correlation, FCD type I appears as mild hyperintensity of the white matter in T2/fluid-attenuated inversion-recovery (FLAIR) sequences with loss of gray/ white matter differentiation. On the other hand, MRI findings in FCD type IIb (dysmorphic neurons with balloon cells) include thickening of the cortex with loss of gray/white matter differentiation as well as a "transmantle sign"-tapering of abnormal white matter signal from the FCD in the cortex to the ventricular surface. About $40 \%$ of patients with FCD type I and approximately $10 \%$ in type II have a normal brain MRI. Thus, a normal brain MRI in a patient with intractable epilepsy does not rule out FCD. Magnetic resonance imaging findings favoring FCD rather than a tumor include cortical gray matter thickening and a transmantle sign. Tubers are not static lesions and there may be dynamic changes over time including calcification and cystic degeneration. The evolution of cystic changes is associated with a TSC2 gene mutation and with having more severe seizures. ${ }^{22}$ More recently, three tuber types $(A, B, C)$ have been distinguished from their MRI features. ${ }^{23}$ However, there is as yet no histopathological classification scheme for tubers; this will represent an important advance in understanding of epileptogenesis in TSC patients. In addition to MRI, functional imaging such as fluorodeoxyglucose-positron emission tomography (FDG-PET) and single photon emission computed tomography (SPECT) can help, particularly in epilepsy localization before surgery. Focal cortical dysplasias typically show focal regional hypometabolism on FDG-PET imaging even in MRI-negative cases. Ictal SPECT detects enhanced cerebral blood flow during the seizures, confirmed with videoelectroencephalogram (EEG) monitoring, and helps with localization in almost half of the patients with FCD. The sensitivity of FDG-PET scan in detecting FCD is 69 to $98 \%$, and for ictal-SPECT it is 48 to $64 \%$.

\section{Focal Cortical Dysplasia: Pathogenesis and Molecular Genetics}

Focal cortical dysplasia and cortical tubers are among the most common pathological substrates associated with medically intractable pediatric epilepsy. ${ }^{1,24}$ Tuberous sclerosis complex is an autosomal dominant, multisystem disorder resulting from mutations in either TSC1 or TSC2, and characterized by a spectrum of neurologic deficits including autism, intellectual disability, and intractable epilepsy. ${ }^{12,25}$ Identification of the TSC1 and TSC2 genes and the links to mammalian target of rapamycin (mTOR) signaling ( - Fig. 2) have provided critical insights into mechanisms of focal MCD, and in fact have provided the paradigm to study other focal MCD subtypes, such as FCDIIb. Tubers form during brain development as a consequence of loss of function mutations in either TSC1 or TSC2, leading to constitutive mTOR activation and altered development of the cerebral cortex. ${ }^{26}$ Numerous studies have shown phosphoactivation of mTORC1 substrates p70S6kinase, 66 , and 4E-BP1 in resected and postmortem TSC tuber samples. ${ }^{27-30}$ Two recent studies showed mTORC1 activation in fetal tubers, ${ }^{17,18}$ suggesting very early activation of mTOR. Renal and pulmonary lesions in TSC follow a "two-hit" mutational model where a somatic inactivating mutation, including loss of heterozygosity or a point mutation in the unaffected allele, is superimposed on the existing germline mutation. Two recent reports suggest that tubers contain both germline and somatic mutations, implying a mechanism of bi-allelic gene inactivation. ${ }^{31,32}$ Mouse models showing abnormal cortical structure have required full Tsc1 or Tsc2 knockout $^{33}$; heterozygous mice do not show significant neuropathological changes.

In contrast to TSC, in FCD, a sporadic disorder with few defined family pedigrees, the pathogenesis remains unknown, but there are some proposed potential pathogenic mechanisms, including somatic gene mutation, or a toxic insult to the developing brain. ${ }^{34-36}$ The pathological similarities between FCDIIb and tubers suggested a mechanistic link between these lesions, and even that FCD represented a sporadic, somatic mosaic form of TSC. Studies have identified TSC1 and TSC2 gene sequence polymorphisms, but not somatic mutations, in FCDIIb. ${ }^{37,38}$ Focal cortical dysplasia can be associated with DEPDC5 mutations (see below), suggesting that syndromic forms of FCD may be identified in association with mutations in mTOR regulatory genes. ${ }^{39}$ A recent study identified somatic trisomy of $1 \mathrm{q} 21-1 \mathrm{q} 44$, a region that encompasses the AKT3 gene, in FCD brain tissue but not in blood or saliva, suggesting a somatic mutational 


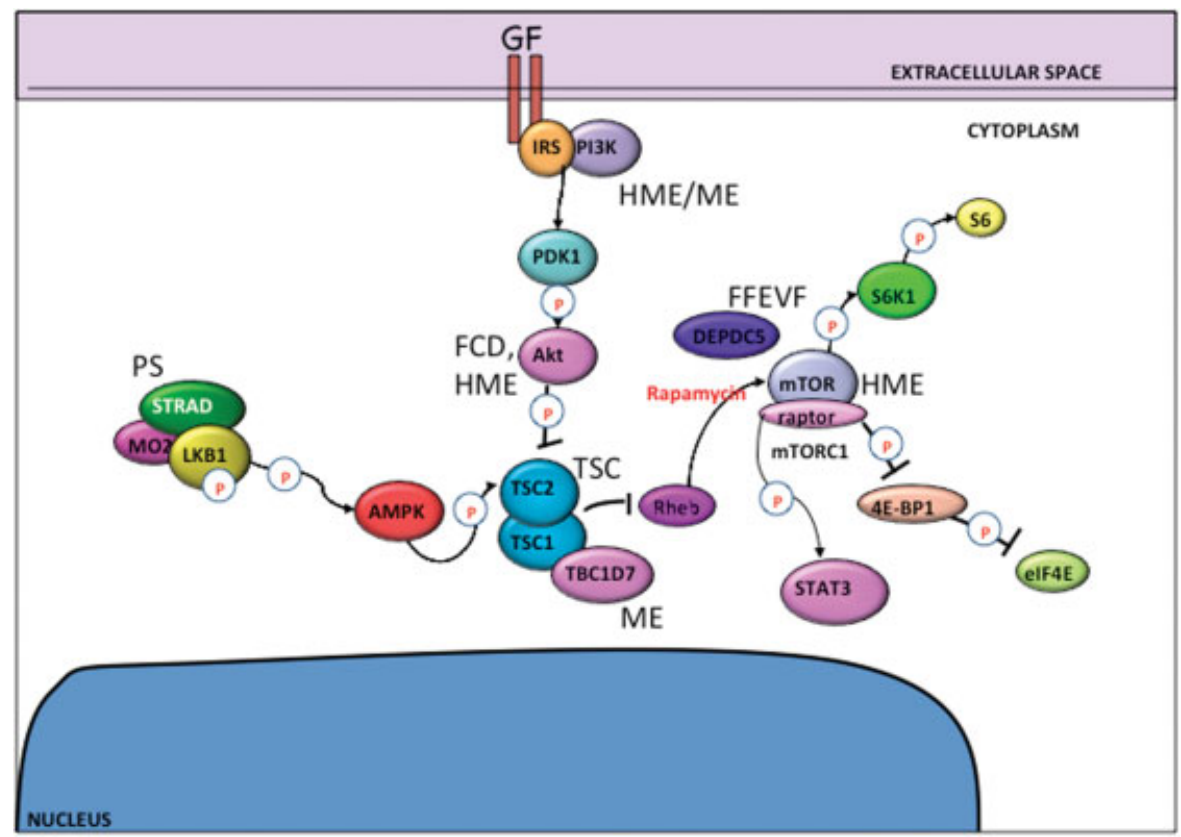

Fig. 2 Schematic depicting mTOR pathway components. Growth factor (GF) receptor-mediated signaling drives the mTOR pathway. Note the mTOR inhibitor rapamycin site of effect. At several key points, individual focal cortical dysplasia (FCD) subtypes are listed next to site of gene mutation in the pathway. For example, tuberous sclerosis complex (TSC) is associated with mutations in either TSC1 or TSC2, FCD with AKT mutations, and FFEVF to DEPDC5 mutations. Hemimegalencephaly has been linked to mutations in PI3K, MTOR. Megalencephaly (ME) has been associated with mutations in PI3K, TBC1D7, and MTOR. Pretzel syndrome (PS) is linked to STRADA mutations and is associated with ME and FCD. For other associations, see text.

mechanism. ${ }^{40}$ Interestingly, another study found FCD type Ia and IIA in a small cohort of patients with mutations in the sodium channel SCN1A. ${ }^{41}$ It is unclear at present how mutations within this channel, which is commonly associated with Dravet syndrome, can alter cortical development; clearly this warrants further investigation.

Enhanced mTOR signaling, evidenced by phospho-p70S6K and phospho-S6 isoforms first identified in FCDIIb, ${ }^{27,28}$ set the stage for subsequent studies (see below), which showed mTOR activation in hemimegalencephaly ${ }^{29,42}$ and ganglioglioma. ${ }^{43}$ In contrast, there is no enhanced p70S6Kinase and S6 phosphorylation (e.g., mTOR activation) in FCD type la or $\mathrm{Ib}^{13}$ The central hypothesis of these studies was that molecular events causing abnormal brain development resulted in mTOR activation, evidenced by hyperphosphorylation of mTOR, p70S6K, and S6 proteins. As in tubers, p70S6K and S6 phospho-isoforms were identified in cells with enlarged somas-dysmorphic neurons and balloon cells in FCDIIb. For example, in tubers or FCDIIb, $>80 \%$ of morphologically defined balloon cells show phospho-S6 labeling. ${ }^{13}$ Furthermore, within any one tuber or FCDIIb specimen, there can be tremendous variability in the number and the distribution of giant cells/balloon cells in the white matter and through the depth of the lesion. Recent studies suggest that a range of epilepsy-associated pathologies, including FCD type III and Rasmussen's encephalitis, may show enhanced mTOR signaling. ${ }^{44}$

Enhanced mTOR signaling is further supported by altered expression of up- and downstream components of the mTOR pathway in FCD. For example, activation of mTOR signals via
HIF $1 \alpha$, initiates vascular endothelial growth factor expression in cortical tubers ${ }^{45}$ and in FCDIIb. ${ }^{46}$ Phosphoactivation of STAT3, a transcription factor regulated by MTOR, is identified in FCDIIb and tubers. ${ }^{27}$ Interestingly, the profiles of phosphorylated proteins in tubers versus FCDIIb are not identical, suggesting potentially different roles for mTOR signaling in the formation of these lesions. For example, phosphoactivation of the upstream cascade proteins p-PDK1 (S241), p-Akt (S473), and p-tuberin (T1462) in FCDIIb is distinct from tubers. ${ }^{47}$ Interestingly, recent studies have suggested possible autophagy induction in FCDIIb and TSC by showing autophagic vacuoles and $\mathrm{p} 62$ expression. ${ }^{48}$ Recent observations suggest that the abnormal activation of mTOR may contribute to apoptosis signaling pathways and premature activation of neurodegeneration cascades in both FCD II and TSC. ${ }^{49}$ For example, tau-immunoreactive neuropil fibrils occur in areas of FCD and hyperphosphorylated tau is detected in pS6 positive dysmorphic neurons.

Two recent studies suggest a viral association for FCD II, ${ }^{50,51}$ which may link to mTOR activation as well as the extant evidence for both innate and adaptive immune responses observed in FCD type II. Human papilloma virus type 16 (HPV16) has been previously associated with dysplasia and cancer of the cervix. The HPV16 oncoprotein E6 is a potent activator of mTOR signaling and was found in FCDIIb specimens by two distinct investigations, ${ }^{50,51}$ although a third investigation did not detect human papilloma virus DNA. ${ }^{52}$ One of these studies also found cytomegalovirus and human herpes virus type $6 .^{51}$ Exogenous expression of E6 in fetal mouse brain causes disorganized cerebral cortical lamination. 
In view of these findings, several future steps will be necessary. First, it must be shown that human papilloma virus can actually infect the brain, for example, neurons or astrocytes, in experimental model systems. Second, analysis of a larger number of cases will be necessary to demonstrate the distribution of FCD specimens with human papilloma virus or other viral species such as cytomegalovirus, which has been previously linked to some types of cortical malformation including pachygyria. ${ }^{34}$ Third, we need to establish the pathogenicity, if any, of human papilloma virus in FCD. Although human papilloma virus is universally associated with cervical cancer, there are distinct subsets of head and neck cancer that either are or are not associated with this virus; thus, stratification of FCD cases may be necessary. Finally, future studies to define the mode of transmission, such as blood borne, must be established. Alternatively, human papilloma virus may have no pathogenic role in FCD.

\section{Familial Focal Epilepsy with Variable Foci}

Recently, mutations were identified in DEPDC5, a gene encoding a protein with tandem N-terminal DEP (disheveled, egl10, pleckstrin) domains; this was in several Australian pedigrees with a clinical phenotype characterized by focal epilepsy arising from distinct lobar locations in different family members. ${ }^{39,53}$ The syndrome, termed familial focal epilepsy with variable foci (FFEVF), is an autosomal dominant disorder associated with epilepsy of varying severity and variable intellectual and neuropsychiatric disorders, including anxiety, depression, and intellectual disability. Some affected patients had "bottom-of-the-sulcus" dysplasias on brain MRI although some patients had other MCD subtypes, such as band heterotopia. ${ }^{54}$ Interestingly, in another report, DEPDC5 mutations were associated with nonlesional focal epilepsies including benign epilepsy with centrotemporal spikes. ${ }^{55}$ DEPDC5 is an important component of the GATOR-1 complex, a modulator of mTOR regulator in response to cellular amino acid levels. ${ }^{56}$ DEPDC5 knockdown leads to enhanced mTORC1 signaling in vitro and thus, FCD in the setting of DEPDC5 mutation has been postulated to be mediated through the mTOR pathway. Further studies will be needed to define the role of DEPDC5 in cortical lamination and epileptogenesis and whether mTOR inhibitors can alter seizure frequency in FFEVF.

\section{CASPR2 and Focal Cortical Dysplasia}

Contactin-associated protein-like 2 (CASPR2) is encoded by CNTNAP2 and functions to cluster voltage-gated potassium channels, such as $\mathrm{K}(\mathrm{v}) 1.1$, at the nodes of Ranvier. Homozygous mutations of CNTNAP2 in Old-Order Amish children were linked to cortical dysplasia, focal epilepsy, and macrocephaly. ${ }^{57}$ In these patients, intractable seizures started in early childhood, after which language regression, hyperactivity, impulsive and aggressive behavior, and intellectual disability appeared in all affected children. Interestingly in the original report, resective surgery did not cure the seizures. Neuropathological examination of resected temporal-lobe specimens showed focal cytoarchitectural abnormalities suggesting a type IIA FCD, as well as widespread astrogliosis and reduced expression of CASPR2. Further studies are clearly warranted to define this mutation outside of the Amish community as well as to define the role of CASPR2 in cortical development.

\section{mTOR, FCD, and Epileptogenesis}

The mechanisms of seizure onset and propagation across the FCD subtypes have not been fully defined. ${ }^{58-60}$ Despite the known association between FCD and intractable seizures, there are many remaining challenges that will help us to understand epileptogenesis (-Fig. 2); these include distinguishing the differential contributions of altered brain structure; the effects of mutations on downstream gene and protein expression; and in the case of FCDIIb, the effects of mTOR hyperactivation on excitability. Although enhanced mTOR signaling is detected in Tsc1, Tsc2, and Pten knockout mouse models associated with spontaneous seizures, hyperactive mTOR signaling also occurs in kainic acid-induced seizures $^{61,62}$ or seizures resulting from electrical brain stimulation $^{63}$ without structural changes in the neocortex or genetic alterations causing increased mTOR activation. Enhanced mTOR activation has been linked to mouse models of infantile spasms ${ }^{64}$ and seizures induced in a hypoxia model lead to increased expression of genes encoding mTOR pathway components. ${ }^{65}$ PI3K- and Akt-dependent mTOR activation has been reported in a rat hippocampal organotypic culture model of posttraumatic epilepsy, and inhibition of PI3K, mTOR, or both (using a dual inhibitor) prevented ictal activity and cell death. ${ }^{66}$ Enhanced mTOR activation is found in human temporal lobe epilepsy specimens. ${ }^{67}$ Mutations in MTOR have been recently identified in epileptic encephalopathies without $\mathrm{MCD},{ }^{68}$ suggesting that enhanced mTOR signaling in the absence of structural abnormalities may lead to epileptogenesis by a yet undefined mechanism. Thus, it appears that while mTOR activation caused by gene mutations in mTOR regulatory elements can induce epileptogenesis, the pathway may also serve as a biomarker for epileptogenic brain tissue.

Human electrophysiological studies have shown the intrinsic epileptogenicity of FCDs. ${ }^{69-71}$ Patients with FCD and TSC have altered numbers and distribution of glutamate and GABA receptor subunits; also electrophysiological recording in surgical FCD specimens in vitro have identified neuronal cellslikely cytomegalic dysmorphic neurons-with hyperexcitable intrinsic membrane properties. ${ }^{71-73}$ In contrast, balloon cells show neither hyperexcitability nor epileptogenicity. Hyperexcitability likely reflects developmental alterations of the balance between excitation and inhibition in the pathogenesis of epileptic focal discharges in FCD including changes in glutamate and GABA receptor expression. ${ }^{74-77}$ Several other studies point to a deregulation of inhibitory synaptic transmission in FCD. ${ }^{78,79}$ Focal cortical dysplasia specimens show downregulation of $G_{A B A} R$ subunits and reduced numbers of inhibitory (GABAergic) neurons, suggesting impaired GABAergic inhibition. ${ }^{19,80-82}$ Electrophysiological studies performed in brain 
slices from FCD tissue show immature GABA receptor-mediated responses; GABA receptor-mediated synchronization appears to be involved in the mechanism leading to in vitro ictal activity in FCD. ${ }^{78,83}$

\section{Clinical Therapeutics}

There have been no clinical trials of mTOR inhibitors such as rapamycin or everolimus for epilepsy associated with FCD. Everolimus reduces the size and volume subependymal giant astrocytomas (SEGA) in TSC. ${ }^{84}$ In a cohort of 26 patients, 16 suffered from seizures, and everolimus treatment modestly improved seizure control in 9 patients. Everolimus also reduces seizure frequency in TSC patients. ${ }^{85}$ Clearly, mTOR inhibition may provide a new target for patients with mTORassociated refractory epilepsy. Polyhydramnios-megalencephaly-symptomatic epilepsy syndrome (Pretzel syndrome) is a neurodevelopmental disorder found in the Old-Order Mennonite community associated with severe epilepsy and caused by mutations in the mTOR regulatory gene STRADA. ${ }^{86}$ A small trial in five children with Pretzel syndrome for between 8 months and 4 years showed that rapamycin (sirolimus in clinical parlance) prevented seizures. ${ }^{87}$ This was the first study to show epilepsy prevention with an mTOR inhibitor and suggested that early treatment could dramatically alter clinical seizure onset. Clearly, we need further consideration of using mTOR inhibitors in other mTOR-associated FCD such as hemimegalencephaly or familial focal epilepsies that are linked to mTOR signaling genes, such as DEPDC5.

\section{Future Considerations}

mTOR hyperactivation during brain development appears to lead to abnormal cortical lamination, cell size, and cell lineage, culminating in intractable epilepsy associated with FCD and TSC, as well as hemimegalencephaly and several other new FCD syndromes. The logical next experimental steps will be to define how each genotype alters mTOR activation, and in turn disrupts cortical development, and to define other factors that contribute to the heterogeneous features of each focal MCD subtype. From a clinical perspective, the mTOR pathway provides new avenues for clinical investigation and for clinical therapeutic trials. In contrast, a uniform mechanism to account for type I FCDs that appear not to be associated with mTOR activation, remains to be identified. We have much work ahead to uncover pathogenic mechanisms for these FCD subtypes, including further investigation into environmental risk factors for FCD, such as viral infection and toxic exposure that may lead to somatic mutations.

\section{Note}

There has been a recent publication reporting somatic mutations in MTOR in FCDIla and FCDIIb (Lim JS, Kim WI, Kang HC, et al. Brain somatic mutations in MTOR cause focal cortical dysplasia type II leading to intractable epilepsy. Nat Med 2015;21(4):395-400).

\section{Acknowledgments}

This work was supported by The National Institute of Neurological Disease and Stroke at the National Institutes of Health (NINDS R01NS082343-01), Citizens United for Research in Epilepsy (CURE).

\section{References}

1 Tassi L, Colombo N, Garbelli R, et al. Focal cortical dysplasia: neuropathological subtypes, EEG, neuroimaging and surgical outcome. Brain 2002;125(Pt 8):1719-1732

2 Aronica E, Crino PB. Epilepsy related to developmental tumors and malformations of cortical development. Neurotherapeutics 2014; 11(2):251-268

3 Sisodiya SM. Malformations of cortical development: burdens and insights from important causes of human epilepsy. Lancet Neurol 2004;3(1):29-38

4 Taylor DC, Falconer MA, Bruton CJ, Corsellis JA. Focal dysplasia of the cerebral cortex in epilepsy. J Neurol Neurosurg Psychiatry 1971;34(4):369-387

5 Crome L. Infantile cerebral gliosis with giant nerve cells. J Neurol Neurosurg Psychiatry 1957;20(2):117-124

6 Sims J. On hypertrophy and atrophy of the brain. Med Chir Trans 1835;19:315-380

7 Bourneville D. Sclérose tubéreuse des circonvolutions cérébrales: idiotie et épilepsie hémiplégique. Arch Neurol (Paris) 1880; 1:81-91

8 Mischel PS, Nguyen LP, Vinters HV. Cerebral cortical dysplasia associated with pediatric epilepsy. Review of neuropathologic features and proposal for a grading system. J Neuropathol Exp Neurol 1995;54(2):137-153

9 Palmini A, Najm I, Avanzini G, et al. Terminology and classification of the cortical dysplasias. Neurology 2004; 62(6, Suppl 3)S2-S8

10 Blümcke I, Thom M, Aronica E, et al. The clinicopathologic spectrum of focal cortical dysplasias: a consensus classification proposed by an ad hoc Task Force of the ILAE Diagnostic Methods Commission. Epilepsia 2011;52(1):158-174

11 Barkovich AJ, Kuzniecky RI, Dobyns WB, Jackson GD, Becker LE, Evrard P. A classification scheme for malformations of cortical development. Neuropediatrics 1996;27(2):59-63

12 Crino PB, Nathanson KL, Henske EP. The tuberous sclerosis complex. N Engl J Med 2006;355(13):1345-1356

13 Orlova KA, Tsai V, Baybis M, et al. Early progenitor cell marker expression distinguishes type II from type I focal cortical dysplasias. J Neuropathol Exp Neurol 2010;69(8):850-863

14 Lamparello P, Baybis M, Pollard J, et al. Developmental lineage of cell types in cortical dysplasia with balloon cells. Brain 2007; 130(Pt 9):2267-2276

15 Hadjivassiliou G, Martinian L, Squier W, et al. The application of cortical layer markers in the evaluation of cortical dysplasias in epilepsy. Acta Neuropathol 2010;120(4):517-528

16 Park SH, Pepkowitz SH, Kerfoot C, et al. Tuberous sclerosis in a 20week gestation fetus: immunohistochemical study. Acta Neuropathol 1997;94(2):180-186

17 Prabowo AS, Anink JJ, Lammens M, et al. Fetal brain lesions in tuberous sclerosis complex: TORC1 activation and inflammation. Brain Pathol 2013;23(1):45-59

18 Tsai V, Parker WE, Orlova KA, et al. Fetal brain mTOR signaling activation in tuberous sclerosis complex. Cereb Cortex 2014; 24(2):315-327 
19 Garbelli R, Meroni A, Magnaghi G, et al. Architectural (Type IA) focal cortical dysplasia and parvalbumin immunostaining in temporal lobe epilepsy. Epilepsia 2006;47(6):1074-1078

20 Simpson SL, Prayson RA. Post-surgical outcome for epilepsy associated with type I focal cortical dysplasia subtypes. Mod Pathol 2014;27(11):1455-1460

21 Colombo N, Salamon N, Raybaud C, Ozkara C, Barkovich AJ. Imaging of malformations of cortical development. Epileptic Disord 2009;11(3):194-205

22 Chu-Shore CJ, Major P, Montenegro M, Thiele E. Cyst-like tubers are associated with TSC2 and epilepsy in tuberous sclerosis complex. Neurology 2009;72(13):1165-1169

23 Gallagher A, Grant EP, Madan N, Jarrett DY, Lyczkowski DA, Thiele EA. MRI findings reveal three different types of tubers in patients with tuberous sclerosis complex. J Neurol 2010;257(8): 1373-1381

24 Krsek P, Maton B, Korman B, et al. Different features of histopathological subtypes of pediatric focal cortical dysplasia. Ann Neurol 2008;63(6):758-769

25 Chu-Shore CJ, Major P, Camposano S, Muzykewicz D, Thiele EA. The natural history of epilepsy in tuberous sclerosis complex. Epilepsia 2010;51(7):1236-1241

26 Orlova KA, Crino PB. The tuberous sclerosis complex. Ann N Y Acad Sci 2010;1184:87-105

27 Baybis M, Yu J, Lee A, et al. mTOR cascade activation distinguishes tubers from focal cortical dysplasia. Ann Neurol 2004;56(4): 478-487

28 Miyata H, Chiang AC, Vinters HV. Insulin signaling pathways in cortical dysplasia and TSC-tubers: tissue microarray analysis. Ann Neurol 2004;56(4):510-519

29 Ljungberg MC, Bhattacharjee MB, Lu Y, et al. Activation of mammalian target of rapamycin in cytomegalic neurons of human cortical dysplasia. Ann Neurol 2006;60(4):420-429

30 Talos DM, Kwiatkowski DJ, Cordero K, Black PM, Jensen FE. Cellspecific alterations of glutamate receptor expression in tuberous sclerosis complex cortical tubers. Ann Neurol 2008;63(4):454-465

31 Crino PB, Aronica E, Baltuch G, Nathanson KL. Biallelic TSC gene inactivation in tuberous sclerosis complex. Neurology 2010; 74(21):1716-1723

32 Qin W, Chan JA, Vinters HV, et al. Analysis of TSC cortical tubers by deep sequencing of TSC1, TSC2 and KRAS demonstrates that small second-hit mutations in these genes are rare events. Brain Pathol 2010;20(6):1096-1105

33 Feliciano DM, Su T, Lopez J, Platel JC, Bordey A. Single-cell Tsc1 knockout during corticogenesis generates tuber-like lesions and reduces seizure threshold in mice. J Clin Invest 2011;121(4): 1596-1607

34 Bosnjak VM, Daković I, Duranović V, Lujić L, Krakar G, Marn B. Malformations of cortical development in children with congenital cytomegalovirus infection - A study of nine children with proven congenital cytomegalovirus infection. Coll Antropol 2011;35(Suppl 1):229-234

35 Crino PB. Focal brain malformations: seizures, signaling, sequencing. Epilepsia 2009;50(Suppl 9):3-8

36 Crino PB. mTOR: a pathogenic signaling pathway in developmental brain malformations. Trends Mol Med 2011;17(12):734-742

37 Becker AJ, Urbach $\mathrm{H}$, Scheffler B, et al. Focal cortical dysplasia of Taylor's balloon cell type: mutational analysis of the TSC1 gene indicates a pathogenic relationship to tuberous sclerosis. Ann Neurol 2002;52(1):29-37

38 Gumbinger C, Rohsbach CB, Schulze-Bonhage A, et al. Focal cortical dysplasia: a genotype-phenotype analysis of polymorphisms and mutations in the TSC genes. Epilepsia 2009;50(6): 1396-1408

39 Baulac S, Ishida S, Marsan E, et al. Familial focal epilepsy with focal cortical dysplasia due to DEPDC5 mutations. Ann Neurol 2015; 77(4):675-683
40 Conti V, Pantaleo M, Barba C, et al. Focal dysplasia of the cerebral cortex and infantile spasms associated with somatic 1q21.1-q44 duplication including the AKT3 gene. Clin Genet 2014

41 Barba C, Parrini E, Coras R, et al. Co-occurring malformations of cortical development and SCN1A gene mutations. Epilepsia 2014; 55(7):1009-1019

42 Aronica E, Boer K, Baybis M, Yu J, Crino P. Co-expression of cyclin D1 and phosphorylated ribosomal 66 proteins in hemimegalencephaly. Acta Neuropathol 2007;114(3):287-293

43 Samadani U, Judkins AR, Akpalu A, Aronica E, Crino PB. Differential cellular gene expression in ganglioglioma. Epilepsia 2007;48(4): 646-653

44 Liu J, Reeves C, Michalak Z, et al. Evidence for mTOR pathway activation in a spectrum of epilepsy-associated pathologies. Acta Neuropathol Commun 2014;2:71

45 Parker WE, Orlova KA, Heuer GG, et al. Enhanced epidermal growth factor, hepatocyte growth factor, and vascular endothelial growth factor expression in tuberous sclerosis complex. Am J Pathol 2011;178(1):296-305

46 Boer K, Troost D, Spliet WG, van Rijen PC, Gorter JA, Aronica E. Cellular distribution of vascular endothelial growth factor $A$ (VEGFA) and B (VEGFB) and VEGF receptors 1 and 2 in focal cortical dysplasia type IIB. Acta Neuropathol 2008;115(6): 683-696

47 Schick V, Majores M, Engels G, et al. Differential Pi3K-pathway activation in cortical tubers and focal cortical dysplasias with balloon cells. Brain Pathol 2007;17(2):165-173

48 Yasin SA, Ali AM, Tata M, et al. mTOR-dependent abnormalities in autophagy characterize human malformations of cortical development: evidence from focal cortical dysplasia and tuberous sclerosis. Acta Neuropathol 2013;126(2):207-218

49 Iyer A, Zurolo E, Spliet WG, et al. Evaluation of the innate and adaptive immunity in type I and type II focal cortical dysplasias. Epilepsia 2010;51(9):1763-1773

50 Chen J, Tsai V, Parker WE, Aronica E, Baybis M, Crino PB. Detection of human papillomavirus in human focal cortical dysplasia type IIB. Ann Neurol 2012;72(6):881-892

51 Liu S, Lu L, Cheng X, Xu G, Yang H. Viral infection and focal cortical dysplasia. Ann Neurol 2014;75(4):614-616

52 Coras R, Korn K, Bien CG, et al. No evidence for human papillomavirus infection in focal cortical dysplasia IIb. Ann Neurol 2015; 77(2):312-319

53 Dibbens LM, de Vries B, Donatello S, et al. Mutations in DEPDC5 cause familial focal epilepsy with variable foci. Nat Genet 2013; 45(5):546-551

54 Scheffer IE, Heron SE, Regan BM, et al. Mutations in mammalian target of rapamycin regulator DEPDC5 cause focal epilepsy with brain malformations. Ann Neurol 2014;75(5):782-787

55 Lal D, Reinthaler EM, Schubert J, et al. DEPDC5 mutations in genetic focal epilepsies of childhood. Ann Neurol 2014;75(5):788-792

56 Bar-Peled L, Chantranupong L, Cherniack AD, et al. A tumor suppressor complex with GAP activity for the Rag GTPases that signal amino acid sufficiency to mTORC1. Science 2013; 340(6136):1100-1106

57 Strauss KA, Puffenberger EG, Huentelman MJ, et al. Recessive symptomatic focal epilepsy and mutant contactin-associated protein-like 2. N Engl J Med 2006;354(13):1370-1377

58 Wong M. Mechanisms of epileptogenesis in tuberous sclerosis complex and related malformations of cortical development with abnormal glioneuronal proliferation. Epilepsia 2008;49(1):8-21

59 Wong M, Crino PB. mTOR and epileptogenesis in developmental brain malformations. In: Noebels JLet al. , eds. Jasper's Basic Mechanisms of the Epilepsies. New York, NY: Oxford University Press; 2012

60 Lasarge CL, Danzer SC. Mechanisms regulating neuronal excitability and seizure development following mTOR pathway hyperactivation. Front Mol Neurosci 2014;7:18 
61 Sha LZ, Xing XL, Zhang D, et al. Mapping the spatio-temporal pattern of the mammalian target of rapamycin (mTOR) activation in temporal lobe epilepsy. PLoS ONE 2012;7(6):e39152

62 Zeng LH, Rensing NR, Wong M. The mammalian target of rapamycin signaling pathway mediates epileptogenesis in a model of temporal lobe epilepsy. J Neurosci 2009;29(21):6964-6972

63 van Vliet EA, Forte G, Holtman L, et al. Inhibition of mammalian target of rapamycin reduces epileptogenesis and blood-brain barrier leakage but not microglia activation. Epilepsia 2012; 53(7):1254-1263

64 Raffo E, Coppola A, Ono T, Briggs SW, Galanopoulou AS. A pulse rapamycin therapy for infantile spasms and associated cognitive decline. Neurobiol Dis 2011;43(2):322-329

65 Theilhaber J, Rakhade SN, Sudhalter J, et al. Gene expression profiling of a hypoxic seizure model of epilepsy suggests a role for mTOR and Wnt signaling in epileptogenesis. PLoS ONE 2013; 8(9):e74428

66 Berdichevsky Y, Dryer AM, Saponjian Y, et al. PI3K-Akt signaling activates mTOR-mediated epileptogenesis in organotypic hippocampal culture model of post-traumatic epilepsy. J Neurosci 2013; 33(21):9056-9067

67 Sosunov AA, Wu X, McGovern RA, et al. The mTOR pathway is activated in glial cells in mesial temporal sclerosis. Epilepsia 2012; 53(Suppl 1):78-86

68 Allen AS, Berkovic SF, Cossette P, et al; Epi4K Consortium; Epilepsy Phenome/Genome Project. De novo mutations in epileptic encephalopathies. Nature 2013;501(7466):217-221

69 Palmini A, Gambardella A, Andermann F, et al. Intrinsic epileptogenicity of human dysplastic cortex as suggested by corticography and surgical results. Ann Neurol 1995;37(4):476-487

70 Matsumoto R, Kinoshita M, Taki J, et al. In vivo epileptogenicity of focal cortical dysplasia: a direct cortical paired stimulation study. Epilepsia 2005;46(11):1744-1749

71 Otsubo H, Iida K, Oishi M, et al. Neurophysiologic findings of neuronal migration disorders: intrinsic epileptogenicity of focal cortical dysplasia on electroencephalography, electrocorticography, and magnetoencephalography. J Child Neurol 2005;20(4):357-363

72 Najm I, Ying Z, Babb T, et al. Mechanisms of epileptogenicity in cortical dysplasias. Neurology 2004; 62(6, Suppl 3):S9-S13

73 Cepeda C, André VM, Levine MS, et al. Epileptogenesis in pediatric cortical dysplasia: the dysmature cerebral developmental hypothesis. Epilepsy Behav 2006;9(2):219-235

74 Ying Z, Babb TL, Mikuni N, Najm I, Drazba J, Bingaman W. Selective coexpression of NMDAR2A/B and NMDAR1 subunit proteins in dysplastic neurons of human epileptic cortex. Exp Neurol 1999; 159(2):409-418

75 Crino PB, Duhaime AC, Baltuch G, White R. Differential expression of glutamate and GABA-A receptor subunit mRNA in cortical dysplasia. Neurology 2001;56(7):906-913

76 Möddel G, Jacobson B, Ying Z, et al. The NMDA receptor NR2B subunit contributes to epileptogenesis in human cortical dysplasia. Brain Res 2005;1046(1-2):10-23

77 Finardi A, Gardoni F, Bassanini S, et al. NMDA receptor composition differs among anatomically diverse malformations of cortical development. J Neuropathol Exp Neurol 2006;65(9): 883-893

78 Cepeda C, André VM, Flores-Hernández J, et al. Pediatric cortical dysplasia: correlations between neuroimaging, electrophysiology and location of cytomegalic neurons and balloon cells and glutamate/GABA synaptic circuits. Dev Neurosci 2005;27(1): 59-76

79 Calcagnotto ME, Paredes MF, Tihan T, Barbaro NM, Baraban SC. Dysfunction of synaptic inhibition in epilepsy associated with focal cortical dysplasia. J Neurosci 2005;25(42): 9649-9657

80 Garbelli R, Munari C, De Biasi S, et al. Taylor's cortical dysplasia: a confocal and ultrastructural immunohistochemical study. Brain Pathol 1999;9(3):445-461

81 Spreafico R, Battaglia G, Arcelli P, et al. Cortical dysplasia: an immunocytochemical study of three patients. Neurology 1998; 50(1):27-36

82 Spreafico R, Tassi L, Colombo N, et al. Inhibitory circuits in human dysplastic tissue. Epilepsia 2000;41(Suppl 6):S168-S173

83 D’Antuono M, Louvel J, Köhling R, et al. GABAA receptor-dependent synchronization leads to ictogenesis in the human dysplastic cortex. Brain 2004;127(Pt 7):1626-1640

84 Krueger DA, Care MM, Holland K, et al. Everolimus for subependymal giant-cell astrocytomas in tuberous sclerosis. N Engl J Med 2010;363(19):1801-1811

85 Krueger DA, Wilfong AA, Holland-Bouley K, et al. Everolimus treatment of refractory epilepsy in tuberous sclerosis complex. Ann Neurol 2013;74(5):679-687

86 Orlova KA, Parker WE, Heuer GG, et al. STRADalpha deficiency results in aberrant mTORC1 signaling during corticogenesis in humans and mice. J Clin Invest 2010;120(5):1591-1602

87 Parker WE, Orlova KA, Parker WH, et al. Rapamycin prevents seizures after depletion of STRADA in a rare neurodevelopmental disorder. Sci Transl Med 2013;5(182):182ra53 